\title{
Applications of Nonlinear Methods to Signal Detection of Time Series
}

\author{
Xingfu Zhong, Liming Lin, Yingxiang Wu \\ Institute of Mechanics, CAS, Beijing, 100190, China \\ xfzhong@imech.ac.cn
}

\begin{abstract}
The analysis of time series from real system is the most direct link between nonlinear theory and real world. If the measure data from nonlinear system are described linearly, useful signal could not found out. The nonlinear methods in this paper, Poincaré map, fractal dimension, and correlation dimension, are introduced to detect chaos phenomena in a system. These nonlinear algorithms can be used to pick up signal characteristics of time series. Some examples are presented to illustrate how to apply these methods in signal detection and engineering signal analysis.
\end{abstract}

\section{Keywords-Nonlinear Methods; Time Series; Signal Analysis}

\section{INTRODUCTION}

The most direct link between nonlinear theory and real world is the analysis of time series from real system. Linear methods interpret all regular structure in data sets, such as a dominant frequency, linear correlations. This means the intrinsic dynamics of system is governed by the linear paradigm that small causes lead to small effects. But a nonlinear system will most likely produce irregular data, and time series consist of random number. If the measure data are properly described linearly, any significant information could not be found. The time series analysis in economics, meteorology, and other fields is very important. And the new prediction algorithms will be found and developed. Some of the techniques as a possible tool in the industry have been considered. Cor M. pointed out a transition from one hydrodynamic regime to another is characterized by a dip in the Kolmogorov entropy ${ }^{[1]}$. Gareth Chaplin carried out the response of the S-statistic to moisture changes resulted from hydrodynamic state changes, not only providing for new means of tracking moisture content within the bed but also allowing for the identification of a specific hydrodynamic state at any point in the drying process ${ }^{[2]}$. Jun $\mathrm{Wu}$ established a global chaos model to predict water quality time series that comprised a small amount of data $^{[3]}$. Shabani pointed out a bifurcation diagram and a Lyapunov exponent plot were also used to investigate the evolution of the response with increasing excitation amplitude $^{[4]}$. Kazuyuki reviewed the research on applications of chaotic dynamics in chaos engineering and previewed a future direction ${ }^{[5]}$. They show that a fractal structure of the strange attractors in the Poincare map is used to qualitatively identify the chaotic responses. The sensitive dependence on initial conditions and a positive Lyapunov exponent practically guarantee chaotic sequences. This paper introduces the nonlinear methods and applications for signal detection. Nonlinear methods can be used to pick up signal characteristic of a data set.

\section{BASIC PRINCIPLE}

\section{A. Poincaré map}

Dynamical system varies with time, so does its status. Therefore, the description of the status space is very important. The space is often composed of a kind of manifold multiplied with space of time coordinate. This manifold is a curved space (including the flat plane space) to indicate all possible situations in system for configuration at certain time. It is called configuration space. Dynamic system is a kind of mapping from the model status space to configuration space. Then, a dynamical system is a mapping.

$$
\vec{\Phi}: \vec{M} \times \vec{R} \rightarrow \vec{M}
$$

where the vector of $M$ is a manifold, which could be an Euclidean space as a flat plane space, $R^{n}$, or a curved space, such as a curved line, a curved plane, or a supercurved plane.

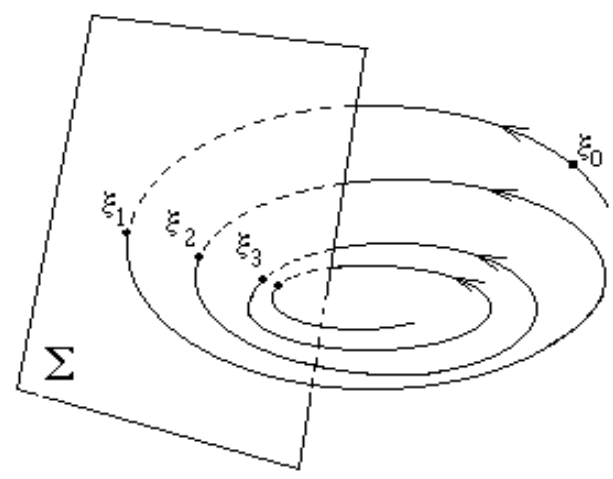

Figure1. Schematic diagram of Poincare section

In an $n$-dimensional space, the (n-1)-dimensional supercurved plane $\Sigma$ is taken from the manifold, as shown in Figure 1 , where $\xi_{0}$ is a point in the n-dimensional space. The trajectory path through $\xi_{0}$ intersects with $\Sigma$ at the point $\xi_{1}$. Then, a series of intersectional points, $\xi_{2}, \xi_{3}$, and so on, would be obtained. Relationship of these points can be explained as $\xi_{n+1}=P_{(\xi n)}$, where $P$ is called Poincare surface of section. The investigation on $P$ is simple because $P$ is the intersection in the (n-1)-dimensional space. By Poincare section, dynamic behaviors of manifold in two, 
three or high dimensional space could be analyzed. Features of Poincare section could be also obtained for manifolds qualitatively. High-dimensional system can be investigated through number of dimensions lowered by Poincare section, as expressed as following,

$$
x_{n+1}=f_{\left(x_{n}\right)}
$$

\section{B. Fractal dimension}

The fractal dimension is a clear sign of different strange attractors. It is the characteristic value of data sets. The following calculation method of fractal dimension bases on Hurst Index. If the time series are $\left\{x_{i}, i=1,2, \cdots, N\right\}$, the deviation of mean value in the time $\tau$ is

$$
x(i, \tau)=\sum_{t=1}^{i}\left[x_{t}-(E(x))_{\tau}\right]=\sum_{t=1}^{i} x_{t}-i(E(x))_{\tau} \quad 1 \leq i \leq \tau
$$

The range is

$$
R(\tau)=\max _{1 \leq i \leq \tau} x(i, \tau)-\min _{1 \leq i \leq \tau} x(i, \tau)
$$

The standard deviation is

$$
\begin{gathered}
S(\tau)=\left\{\frac{1}{\tau} \sum_{i=1}^{\tau}\left[x_{i}-(E(x))_{\tau}\right]^{2}\right\}^{1 / 2} \\
{[E(x)]_{\tau}=\left(\sum_{i=1}^{\tau} x_{i}\right) / \tau}
\end{gathered}
$$

Here $x_{i}$ is the measure data set. A new data set $(\tau, \operatorname{R}(\tau) / S(\tau))$ from $x_{i}$ can be created, and the data points in double logarithmic coordinate system is drawn. The slope of line $(\operatorname{Ln}(\tau), \operatorname{Ln}(R(\tau) / S(\tau)))$ fitted by least squares fit is Hurst Index $(H)$. The fractal dimension $D$ is

$d-H$. Here $d$ is dimension of Euclidean space.

\section{C. orrelation dimension}

The correlation dimension is the response of a system state. The system state is a point in phase space. The phase space can be reconstructed from time series. Therefore, the first step is the reconstruction of phase space.

By assuming that time series $x_{(i)}$ are $\left\{x_{1}, x_{2}, x_{3}, \ldots, x_{N}\right\}$, the vector $y$ with dimension $m$ is reconstructed. If $m \geq 2 D+1$, where $D$ is dimension of the strange attractor, $y_{(i)}=\left\{x_{i}, x_{i+\tau}, x_{i+2 \tau}, \ldots, x_{i+(m-1)}\right\}, i=1,2,3, \ldots \varepsilon \varepsilon$ is defined as a given distance in particular norm. If a pair of $y_{(i)}$ are closer than $\varepsilon$, the basic formula (to be modified for practical application) is given by

$$
C_{(\varepsilon)}=\frac{1}{M^{2}} \sum_{i, j=1}^{m} H\left(\varepsilon-\left|y_{i}-y_{j}\right|\right)
$$

where $H$ is Heaviside step function,

$$
H_{(x)}=\left\{\begin{array}{l}
1, x>0 \\
0, x \leq 0
\end{array}\right.
$$

Correlation dimension is defined as

$$
D_{C}=\lim _{\varepsilon \rightarrow 0} \log C_{(\varepsilon)} / \log (\varepsilon)
$$

The Dc implies dynamic information of a nonlinear dynamic system. The minimum Dc expresses the minimum number of state variable used to describe a system.

\section{APPLICATIONS}

\section{A. Poincare map}

Figure 2 is an application of quickly detecting whether the structural VIV is occurred from time series of measured signals ${ }^{[6]}$. Figure $2 \mathrm{a}$ is Poincare map of $0^{\#}$ cylinder oscillation. The amplitude of oscillation reaches up to the maximum at the velocity of $5 \mathrm{~cm} / \mathrm{s}$ and $10 \mathrm{~cm} / \mathrm{s}$. Figure $2 \mathrm{~b}$ is Poincare map of $5^{\#}$ cylinder oscillation. With the velocity increasing up to 10,13 and $15 \mathrm{~cm} / \mathrm{s}$, the elliptic shape appeases on Poincare map, which indicates the system enters into a different periodic orbit. The amplitude of oscillation reaches up to the maximum at velocity of $13 \mathrm{~cm} / \mathrm{s}$. This experiment shows that Poincare map is quick and reliable as a new means of detection of VIV for engineering.

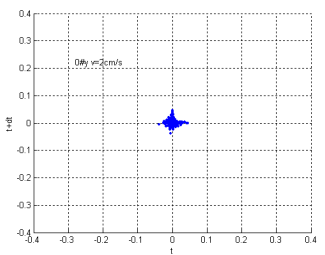

(a) $2 \mathrm{~cm} / \mathrm{s}$

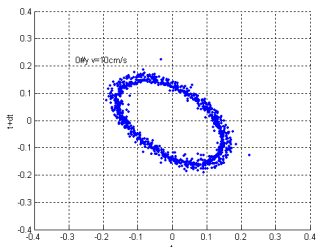

(c) $10 \mathrm{~cm} / \mathrm{s}$

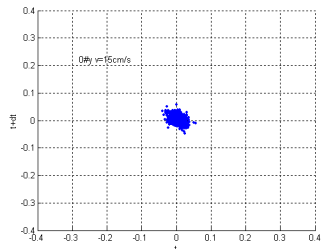

(e) $15 \mathrm{~cm} / \mathrm{s}$

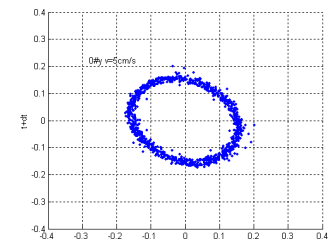

(b) $5 \mathrm{~cm} / \mathrm{s}$

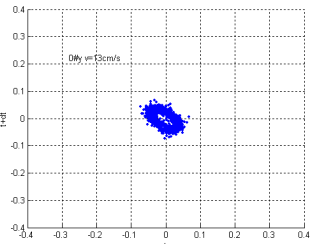

(d) $13 \mathrm{~cm} / \mathrm{s}$

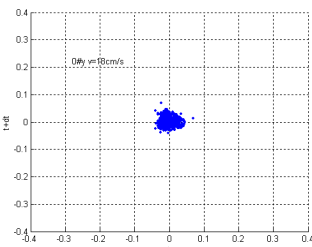

(f) $18 \mathrm{~cm} / \mathrm{s}$ a. The Poincare map of $0^{\#}$ cylinder oscillation 


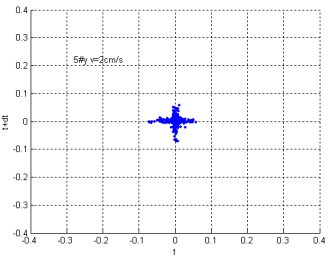

(a) $2 \mathrm{~cm} / \mathrm{s}$

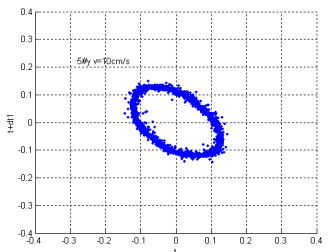

(c) $10 \mathrm{~cm} / \mathrm{s}$

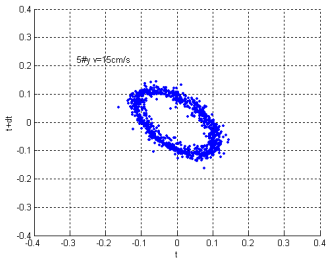

(e) $15 \mathrm{~cm} / \mathrm{s}$
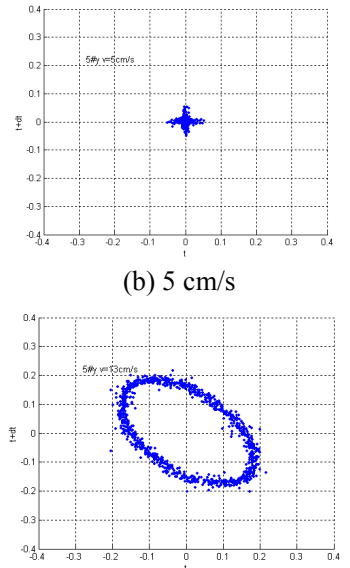

(d) $13 \mathrm{~cm} / \mathrm{s}$

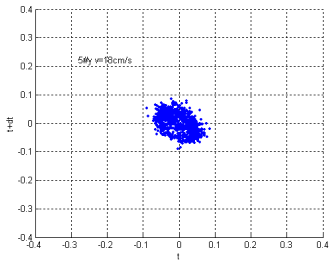

(f) $18 \mathrm{~cm} / \mathrm{s}$ (b) $5 \mathrm{~cm} / \mathrm{s}$

b. The Poincare map of $5 \#$ cylinder oscillation

Figure2. Poincare sections of non-dimensional amplitude of vertical oscillation for $0 \#$ and $5 \#$ cylinder at different velocities

\section{B. Fractal dimension}

Flow patterns can be distinguished by using the fractal dimension $^{[7]}$. Figure 3 shows three flow patterns divided by fractal dimension. The data are the time series of measured water holdup wave. When flow pattern changes from $\mathrm{A}$ to $\mathrm{C}$, the fractal dimension increases gradually. In pattern $\mathrm{A}$ the fractal dimension $\mathrm{d}$ ranges from 1.051 to 1.216 , in patten $\mathrm{B}$ from 1.217 to 1.418 , and in patten $\mathrm{C}$ with 1.25 . In the same pattern, the fractal dimension of flow pattern has a small difference. Diferent patterns have different levels of fractal dimension.

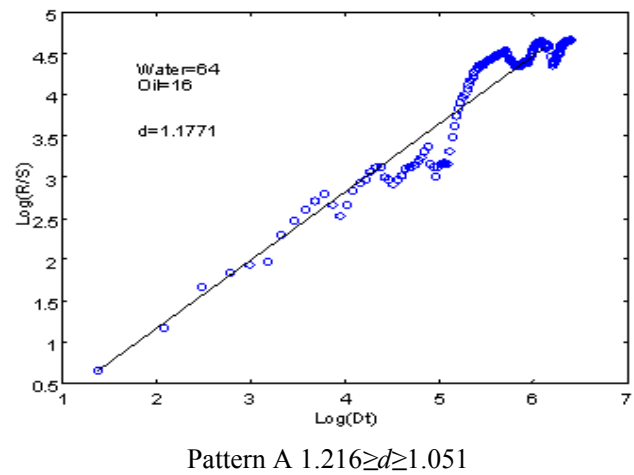

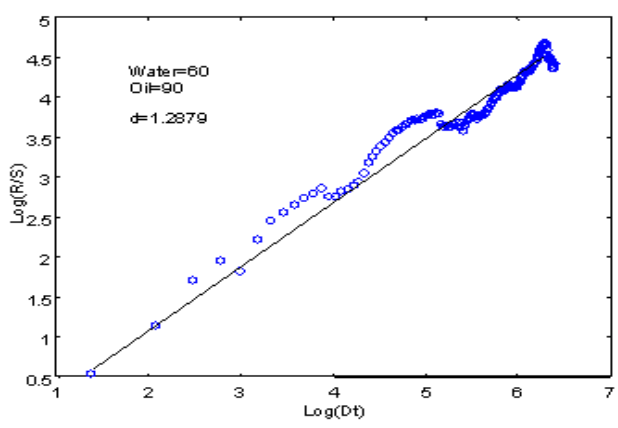

Pattern B $1.418 \geq d \geq 1.217$

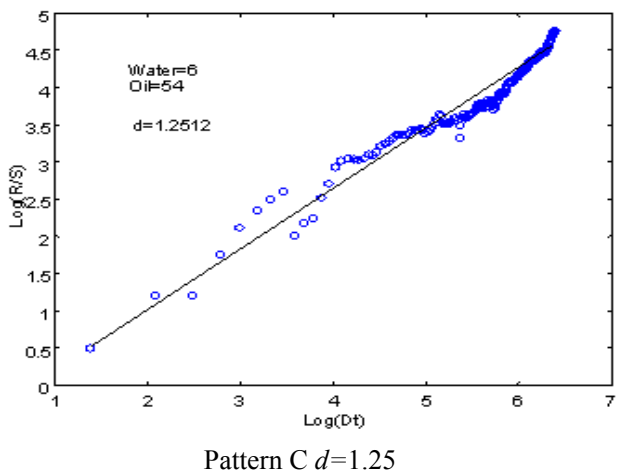

Figure 3. The fractal dimension d of water holdup wave

\section{Correlation dimension}

The correlation dimension is another way to recognize flow patterns ${ }^{[7]}$. Figure 4 is the result of estimated correlation dimension. The data are collected from measured water holdup wave. In pattern A the correlation dimension Dc ranges from 1.657 to 1.966 , in patten $\mathrm{B}$ from 1.9714 to 2.726 , and in patten $\mathrm{C}$, Dc is 3.175 . The result shows that correlation dimension is available to distinguish different flow patterns clearly.The correlation dimension of water holdup wave can play an important role in recognizing flow patterns. And the correlation dimension is more effective than the fractal dimension to recognize flow pattern. The correlation dimension is a good indicator of flow pattern recognization.

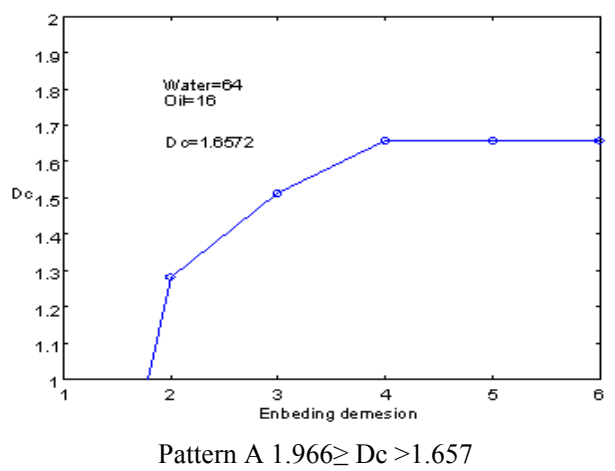




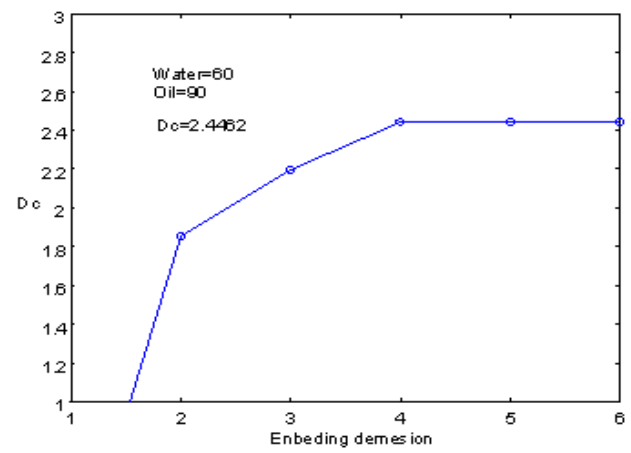

Pattern B 2.726 $\geq$ Dc $>1.9714$

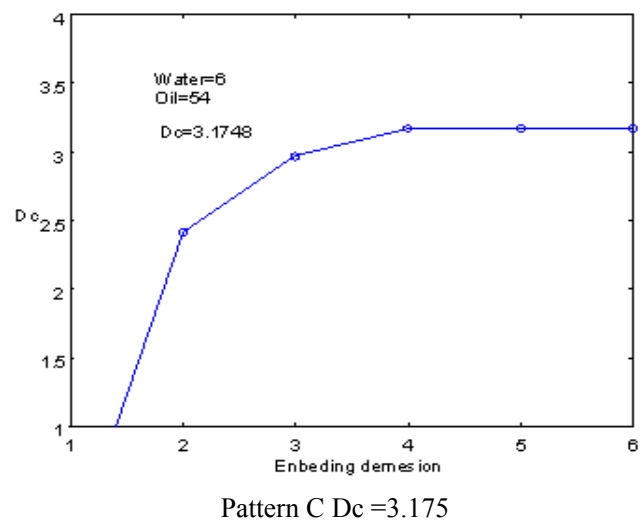

Figure 4. The correlation dimension Dc of water holdup wave

\section{CONCLUSION}

The nonlinear methods have been introduced and explained. Three different examples are illustrated: Poincaré map, fractal dimension, and correlation dimension. Nonlinear methods can be used to pick up the signal characteristic of a data set. It will become a useful tool for signal detection and engineering signal analysis.

\section{REFERENCES}

[1] Cor M. van den Bleeka, Marc-Olivier Coppensa. Science Vol.57 (2002), p. 4763

[2] Gareth Chaplin, Todd Pugsley, Conrad Winters. Powder Technology Vol.142 (2004),p.110

[3] Jun Wu, Jian Lu, Jiaquan Wang. Environmental Modelling \& Software Vol. 24 (2009), p.632

[4] R. Shabani. S, Tariverdilo, H. Salarieh. Transactions of the ASME. Vol. 7(2012), 021012-1

[5] Kazuyuki Aihara. IUTAM Symposium on 50 Years of Chaos. Procedia IUTAM 5 (2012), p. 199

[6] Xingfu Zhong, Liming Lin, Yingxiang Wu. IEEE CFP1154K-PRT (2011), p.2752

[7] Xingfu Zhong, Zhiyao Huang, Pengjv Lu. PETROLEI SINICA. Vol. 22 (2001), p.89 . (in Chinese) 